\title{
AN EULERIAN DESCRIPTION OF FLUIDS CONTAINING VISCO-ELASTIC PARTICLES*
}

\author{
CHUN LIU ${ }^{\dagger}$ AND NOEL J. WALKINGTON ${ }^{\ddagger}$
}

\begin{abstract}
Equations governing the flow of fluid containing visco-hyperelastic particles are developed in an Eulerian framework. The novel feature introduced here is to write an evolution equation for the strain. It is envisioned that this will simplify numerical codes which typically compute the strain on Lagrangian meshes moving through Eulerian meshes. Existence results for the flow of linear visco-hyperelastic particles in a Newtonian fluid are established using a Galerkin scheme.
\end{abstract}

Key words. fluid solid mixtures, visco-hyperelastic particles, Eulerian description

1. Introduction. When modeling physical systems that contain both fluid and solid particles one is always confronted with the dilemma that fluids are naturally described using the Eulerian (spatial) description yet solids are naturally described in a Lagrangian (referential) frame. From an analysts point of view this decoupling of the problem presents significant technical challenges. The equation for the fluid takes place on a time dependent domain (the region not currently occupied by the solid), and the regularity of the solution is usually low so that the change of coordinates relating the two descriptions is not smooth. The numerical simulation of such systems is similarly plagued. If the solid particles are represented by a Lagrangian mesh it is necessary to interpolate their image into the Eulerian mesh, and this is expensive and degrades accuracy $[12,26]$. Moreover, the absence of a satisfactory theory for the underlying equations undermines the analysis of these algorithms.

We consider the equations for the flow of a fluid containing visco-hyperelastic solid particles. We pose the basic equations in a purely Eulerian description; numerical simulation of such a system will only require a single mesh for the Eulerian domain. The system of equations we propose contains the classical visco-hyperelasticity equations for which there is no satisfactory theory of existence and uniqueness $[7,16]$. However, we consider an approximation for which it is possible to develop a reasonable existence theory. This approximation corresponds to an appropriate description of visco-hyperelasticity for the solid particles for which the strains but not the rotations are small. This simplified system should provide a good model problem for the analysis and comparison of various numerical algorithms.

\footnotetext{
*SUBMITTED ARCHIVE FOR RATIONAL MECHANICS AND ANALYSIS, 12/00

${ }^{\dagger}$ Department of Mathematics, Pennsylvania Sate University, State College, PA 16802, liu@math.psu.edu. Supported in part by National Science Foundation Grant DMS-9972040.

‡Department of Mathematics, Carnegie Mellon University, Pittsburgh, PA 15213, noelw@cmu.edu. Supported in part by National Science Foundation Grants DMS-9973285 and CCR-9902091. This work was also supported by the NSF through the Center for Nonlinear Analysis.
} 
Elastic materials are typically described in Lagrangian coordinates since the stress depends upon the deformation gradient $F$ from a fixed configuration, and $F$ is not immediately available in an Eulerian description. We circumvented this by writing an evolution equation for $F$, our equation (2.5). Our description also utilizes a "phase" variable $\phi$ equal to \pm 1 in the fluid/solid regions. This approach has been used in the past for the simulation of the flow of immiscible fluids [5, 17, 18, 25, 24], and essentially circumvents the "mapping" problem encountered by the numerical analysts described in the first paragraph.

The interaction of Eulerian and Lagrangian descriptions is ubiquitous in the plasticity literature $[1,2]$. Classically numerical computations are based upon a Lagrangian mesh [1], and the large plastic deformations can result in tangled meshes and ill-conditioned systems of equations. The computations in [11] utilize an Eulerian description which contains a free-boundary problem to determine the surface of the solid. Since the problem in [11] was one-dimensional it was relatively easy to track the motion of the free surface through the mesh; however, this would seem a difficult task in multiple dimensions where, for example, topological changes could occur due to contact. This problem of determining the location of the particles (and their surfaces) is circumvented here by exploiting a phase variable to track them.

1.1. Notation. We adopt the standard notation of continuum mechanics [14]. $X \in$ $\mathbb{R}^{d}$ is the material description, $x=\chi(X, t)$ is the position of particle $X$ at time $t$, and the velocity is given by $v=\dot{x}$ where the dot indicates the partial derivative with respect to time with $X$ fixed (the material or convective derivative). In the Eulerian description $(x, t)$ the chain rule gives $\dot{g}=g_{t}+v \cdot \nabla g$ where $\nabla$ is the gradient in the $x$ variables. Classical mechanics assumes that $\chi: \mathbb{R}^{d} \rightarrow \mathbb{R}^{d}$ is a diffeomorphism and the deformation gradient $F=\left[\partial x_{i} / \partial X_{\alpha}\right]$ is the Jacobian of this mapping and has $J=\operatorname{det}(F)>0$. Below we will consider incompressible materials for which $J=1$. If the elastic part of the stress of a solid particle depends only upon the deformation gradient $F$, it must take the form $(1 / J) \mathcal{D} \mathcal{W}(F) F^{T}$ where $\mathcal{W}: \mathbb{R}^{d \times d} \rightarrow \mathbb{R}$ is the strain energy function and $(\mathcal{D W})_{i \alpha}=\partial \mathcal{W} / \partial F_{i \alpha}$ is the Piola Kirchhoff stress tensor. The strain energy function must satisfy $\mathcal{W}(R U)=\mathcal{W}(U)$ for all proper orthogonal matrices (i.e. $R R^{T}=I$, $\operatorname{det}(R)>0$ ) and hence $\mathcal{D} \mathcal{W}(R U)=R \mathcal{D} \mathcal{W}(U)$. If $F=R U$ with $U=U^{T}$ represents the polar decomposition of the deformation gradient it follows that the stress becomes $(1 / J) R \mathcal{D} \mathcal{W}(U) U R^{T}$. When the Piola Kirchhoff stress tensor is the gradient of a strain energy function, as above, the material is called hyperelastic.

Classical linear elasticity assumes that the displacement $u=x-X$ is small so that $F=I+H$, where $H=\nabla_{X} u$ is small. In this situation the polar decomposition is, to first order, $F \simeq\left(I+H_{\text {skew }}\right)\left(I+H_{\text {sym }}\right)$ where $H_{\text {skew }}$ and $H_{\text {sym }}$ are the skew and symmetric parts of $H$. If the "residual stress" $\mathcal{D W}(I)$ vanishes, then, to first order, the stress becomes $\mathbb{C}\left(H_{\text {sym }}\right)$ where $\mathbb{C}: \mathbb{R}^{d \times d} \rightarrow \mathbb{R}^{d \times d}$ is the second derivative of $\mathcal{W}$ at the identity. Symmetry of the stress tensor implies $\mathbb{C}$ is symmetric in the sense that $\mathbb{C}(A) \cdot B=A \cdot \mathbb{C}(B)$ where $A \cdot B=\sum_{i j} A_{i j} B_{i j}$ is the Frobenius inner product. It 
is traditional to assume that $\mathcal{W}$ assumes its minimum value at $I$ and that the second derivative is strictly positive definite, that is $\mathbb{C}(A) \cdot A \geq c|A|^{2}$, where $c>0$ and $|A|^{2}=A \cdot A$ is the Frobenius norm. In this situation $\mathbb{C}$ induces an inner product $(., .)_{\mathbb{C}}$ on $\mathbb{R}^{d \times d}$.

Below $\Omega \subset \mathbb{R}^{d}$ will denote a bounded domain with Lipschitz boundary. Standard notation is adopted for the Lebesgue spaces, $L^{p}(\Omega)$, and the Sobolev spaces, $W^{m, p}(\Omega)$ or $H^{m}(\Omega)$. The dual exponent to $p$ will be denoted by $p^{\prime}, 1 / p+1 / p^{\prime}=1$. Solutions of various evolution problems will be functions from $[0, T]$ into these spaces, and we adopt the usual notion, $L^{2}\left[0, T ; H^{1}(\Omega)\right], C\left[0, T ; H^{1}(\Omega)\right]$, etc. to indicate the temporal regularity of such functions. For vector or matrix valued quantities, such as the velocity $v$ or deformation gradient $F$, we write $v \in L^{2}(\Omega), F \in L^{2}(\Omega)$, to indicate that each component lies in the specified space. Strong convergence of a sequence will be indicated as $v_{n} \rightarrow v$, and weak convergence by $v_{n} \rightarrow v$.

Divergences of vectors and matrices are denoted $\operatorname{div}(v)=v_{i, i}$ and $\operatorname{div}(T)_{j}=T_{i j, j}$, and gradients of vector valued quantities are interpreted as matrices, $(\nabla v)_{i j}=v_{i, j}$. Here indices after the comma represent partial derivatives and the summation convention is used. The symmetric part of the velocity gradient (stretching tensor) is written as $D(u)$, and the skew part written as $W(v)$ (spin tensor). Inner products of vectors $v, w \in \mathbb{R}^{d}$ are written as $v . w$ and the Frobenius inner product of two matrices $A$, $B \in \mathbb{R}^{d \times d}$ is denoted by $A \cdot B=\sum_{i, j} A_{i j} B_{i j}$. We frequently use the elementary identities $A B \cdot C=A \cdot C B^{T}=B \cdot A^{T} C$.

1.2. Outline. In the next section we present an Eulerian description of a system consisting of a fluid containing particles with a focus on the situation where the fluid is Newtonian and the particles are visco-hyperelastic. As stated previously, currently there is no satisfactory existence theory for solutions of the viscoelastic equations, so in Section 3 we develop approximate equations which model situations for which the strain in the solid is small. The final section establishes existence of solutions of the approximate equations. The proof of existence draws heavily from the ideas developed in DiPerna and Lions [8] and Lions [22] where convection equations and fluids with variable density are studied.

2. Eulerian Description of Fluid/Solid Particles. Let $\Omega \subset \mathbb{R}^{d},(d=2$ or 3$)$ be a domain with boundary $\partial \Omega$. We consider a model where $\Omega$ is filled with a fluid containing solid particles and write

$$
\bar{\Omega}=\bar{\Omega}_{f}(t) \cup \bar{\Omega}_{s}(t)
$$

where $\Omega_{f}$ is the region occupied by the fluid and $\Omega_{s}$ is the region occupied by the solid particles, each of which may be disconnected.

Formulae for the density, stress tensor, etc. at a point $(x, t)$ will depend upon whether fluid is currently at $x,\left(x \in \Omega_{f}(t)\right)$, or a solid particle is currently at the position $x$. For 
example, for incompressible materials,

$$
\rho= \begin{cases}\rho_{f} & \text { in the fluid, and } \\ \rho_{s} & \text { in the solid, }\end{cases}
$$

$\rho_{f}, \rho_{s} \in \mathbb{R}^{+}$, and a similar formula holds for the stress tensor $T$. A convenient way to write this is to introduce a phase function $\phi(x, t)$ equal to +1 in the fluid and -1 in the solid,

$$
\phi(x, t)= \begin{cases}+1 & x \in \Omega_{f}(t), \\ -1 & x \in \Omega_{s}(t) .\end{cases}
$$

We think of the level set $\phi=0$ as the solid/fluid interface. Then

$$
\rho=\frac{1+\phi}{2} \rho_{f}+\frac{1-\phi}{2} \rho_{s} \equiv \chi_{f} \rho_{f}+\chi_{s} \rho_{s}
$$

where $\chi_{f}$ and $\chi_{s}$ are the characteristic functions of the fluid an solid regions respectively.

Notice that when expressed in Lagrangian coordinates $\phi$ is independent of time, $\phi(x(X, t), t)=$ $\Phi(X)$, so $\dot{\phi}=0$ or, equivalently,

$$
\phi_{t}+v \cdot \nabla \phi=0
$$

in an Eulerian frame $\left(\nabla=\nabla_{x}\right)$. Since $\phi$ is discontinuous this equation must be interpreted in the usual weak sense, that is,

$$
\int_{0}^{T} \int_{\Omega} \phi\left(\psi_{t}+v \cdot \nabla \psi+\operatorname{div}(v) \psi\right)=\left.\int_{\Omega} \phi \psi\right|_{0} ^{T}+\int_{0}^{T} \int_{\partial \Omega} \phi \psi v \cdot n
$$

for smooth functions $\psi$. In order to avoid multiplying distributions it may be necessary to require the velocity $v$ to have some regularity. We will assume that the fluid is viscous so that it sticks to the particles. In this situation classical solutions have $v$ continuous throughout $\Omega$.

Balance of Mass. Balance of mass requires that

$$
\rho_{t}+\operatorname{div}(\rho v)=0 \text {. }
$$

Since $\rho$ is not continuous: this equation is required to hold in the weak sense:

$$
\int_{0}^{T} \int_{\Omega} \rho\left(\psi_{t}+v \cdot \nabla \psi\right)=\left.\int_{\Omega} \rho \psi\right|_{0} ^{T}+\int_{0}^{T} \int_{\partial \Omega} \psi \rho v \cdot n .
$$

When the velocity field is divergence free, $\operatorname{div}(v)=0$, the equations for balance of mass and convection of $\phi$ are identical. In fact, since $\rho=(1 / 2)\left(\rho_{f}-\rho_{s}\right) \phi+\left(\rho_{f}+\rho_{s}\right) / 2$ is an affine function of $\phi$, the weak form of balance of mass is satisfied whenever the weak statement for $\phi$ holds. To observe this, notice that if $\phi$ satisfies the weak form of the 
convection equation, then so too does $\alpha \phi+\beta$ for any $\alpha, \beta \in \mathbb{R}$ (assuming that $v$ satisfies some minimal regularity), and hence so too does $\rho$.

The observation that affine functions of $\phi$ satisfy the same weak statement as $\phi$ is a special case of a theorem by Liouville and a more general result by DiPerna and Lions [8]. Under suitable regularity assumptions on $v$, any continuous function of the form $\beta(\phi)$ with $\beta: \mathbb{R} \rightarrow \mathbb{R}$ will also be a weak solution of the convection equation.

Balance of Momentum. We write balance of momentum in a weak form to avoid having to explicitly introduce tractions across the fluid solid interfaces. This weak equation represents balance of momentum in situations for which the velocity is smooth (at least continuous) and the density and stresses possibly discontinuous:

$$
\int_{\Omega} \rho v_{t} \cdot w+\rho(v \cdot \nabla) v \cdot w+T \cdot D(w)=\int_{\Omega} \rho f \cdot w
$$

for smooth vector fields $w: \Omega \rightarrow \mathbb{R}^{d}$ vanishing on $\partial \Omega$. Here $T=T^{T}$ is the Cauchy stress tensor, and $D(w)=\left(\nabla w+(\nabla w)^{T}\right) / 2$ is the stretching tensor for the field $w$.

The constitutive equation for the stress tensor differs for fluids and solids, so we write

$$
T=\chi_{f} T_{f}+\chi_{s} T_{s}
$$

We consider the situation where $T_{f}$ depends upon the stretching tensor $D(v)$, while $T_{s}$ depends additionally upon the deformation gradient $F$. The prototypical situation of an incompressible Navier Stokes fluid containing incompressible visco-hyperelastic particles would have

$$
T_{f}=-p I+\mu_{f} D(v), \quad \text { and } \quad T_{s}=-p I+\mu_{f} D(v)+\mathcal{D} \mathcal{W}(F) F^{T} .
$$

Here $\mathcal{W}: \mathbb{R}^{d \times d} \rightarrow \mathbb{R}$ is the strain-energy function, and $p$ is the pressure.

Computing the Deformation Gradient. We finally address the question of how to compute the deformation gradient tensor. An application of the chain rule gives an Eulerian description,

$$
\dot{F}=\frac{\partial}{\partial t} \frac{\partial x}{\partial X}(X, t)=\frac{\partial v}{\partial X}(X, t)=\frac{\partial v}{\partial x}(x, t) \frac{\partial x}{\partial X}(X, t),
$$

which we write as

$$
F_{t}+(v \cdot \nabla) F=(\nabla v) F
$$

the product on the right being a matrix product. Notice that in order to compute $T$, we need only compute $F$ in the solid where $\phi=-1$; in fact, $F$ would become a rather wild function in the fluid. Observe that if we define $F_{s}=\chi_{s} F$, then since $\dot{\chi}_{s}=0$ we obtain

$$
F_{s_{t}}+(v \cdot \nabla) F_{s}=(\nabla v) F_{s} .
$$

Clearly solutions of this equation are those obtained simply by multiplying the initial data for equation $(2.5)$ by $\chi_{s}(0)$; in effect, specifying $F=0$ in the fluid. 
2.1. Summary. The equations for the evolution of an incompressible Newtonian fluid carrying incompressible visco-hyperelastic particles are

$$
\begin{gathered}
\int_{\Omega} \rho\left(v_{t}+(v \cdot \nabla) v\right) \cdot w+p d i v(w)+\mu D(v) \cdot D(w)+\chi_{s} \mathcal{D} \mathcal{W}(F) F^{T} \cdot D(w)=\int_{\Omega} \rho f \cdot w \\
\nabla \cdot v=0, \\
\phi_{t}+v \cdot \nabla \phi=0,
\end{gathered}
$$

and

$$
F_{t}+(v \cdot \nabla) F=(\nabla v) F
$$

The characteristic functions $\chi_{f}, \chi_{s}$ are computed from $\phi$ as $(1 \pm \phi) / 2$ and the density and viscosity are computed as $\rho=\rho_{f} \chi_{f}+\rho_{s} \chi_{s}$ and $\mu=\mu_{f} \chi_{f}+\mu_{s} \chi_{s}$ with $\rho_{f}, \rho_{s}$ and $\mu_{f}, \mu_{s}$ each non-negative.

Initial values are specified for the velocity $\left.v\right|_{t=0}=v_{0}$ and the phase function $\left.\phi\right|_{t=0}=\phi_{0}$. Typically the initial deformation gradient is the identity on the solid particles, and set arbitrarily to zero in the fluid, $F_{0}=\chi_{s}(0) I$. If non-zero Dirichlet boundary data on the velocity is specified it is necessary to specify $\phi$ and $F$ on those portions of $\partial \Omega$ for which $v . n<0$; that is, specify if fluid or solid particles are entering the domain and for the solid particles it is necessary to specify their deformation gradient (we set $F=0$ in the fluid). While it is easy to specify traction boundary conditions for the momentum equation, this can give rise to technical problems since it is possible that the portion of $\partial \Omega$ where $v . n<0$ varies with time in an implicit fashion, and this is where boundary values for $\phi$ and $F$ are specified. Also, it is not clear what traction to specify on interior portions of particles emanating from the domain.

2.2. Balance of Energy. As with the density we write $\mu=\mu_{f} \chi_{f}+\mu_{s} \chi_{s}$ for the viscosity and will assume that $\mu_{f}, \mu_{s}>0$. For ease of exposition we will consider the situation where $v$ vanishes on $\partial \Omega$ (Dirichlet boundary conditions):

$$
\left.v\right|_{\partial \Omega}=0
$$

Formal calculations are used to develop an energy estimate. Put $w=v$ in the momentum equation and select $\psi=|v|^{2} / 2$ in the weak statement of the balance of mass $(2.2)$ to obtain

$$
\int_{\Omega} \rho\left(|v|^{2} / 2\right)_{t}+\rho v \cdot \nabla\left(|v|^{2} / 2\right)+\mu|D(v)|^{2}+\chi_{s} \mathcal{D} \mathcal{W}(F) \cdot(\nabla v) F=\int_{\Omega} \rho f . v
$$

and

$$
\int_{0}^{T} \int_{\Omega} \rho\left(\left(|v|^{2} / 2\right)_{t}+v \cdot \nabla\left(|v|^{2} / 2\right)\right)=\left.\int_{\Omega} \rho\left(|v|^{2} / 2\right)\right|_{0} ^{T} .
$$


The condition $\operatorname{div}(v)=0$ was used to eliminate the term involving the pressure, and the Dirichlet boundary data on $v$ eliminated the boundary term in the weak statement of balance of mass. Adding these equations gives

$$
\left.\int_{\Omega} \rho\left(|v|^{2} / 2\right)\right|_{0} ^{T}+\int_{0}^{T} \int_{\Omega} \mu|D(v)|^{2}+\chi_{s} \mathcal{D} \mathcal{W}(F) \cdot(\nabla v) F=\int_{\Omega} \rho f . v .
$$

To accommodate the term involving the elastic energy, recall equation (2.5) satisfied by the deformation gradient: $F_{t}+(v \cdot \nabla) F=(\nabla v) F$. Since $\dot{\chi}_{s}=0$ it follows that

$$
\chi_{s} \mathcal{D} \mathcal{W}(F) \cdot(\nabla v) F=\chi_{s}\left(\mathcal{W}(F)_{t}+(v \cdot \nabla) \mathcal{W}(F)\right)=\left(\chi_{s} \mathcal{W}(F)\right)_{t}+(v \cdot \nabla)\left(\chi_{s} \mathcal{W}(F)\right)
$$

The Dirichlet data assumed for $v$ then allows us to conclude that

$$
\int_{0}^{T} \int_{\Omega} \chi_{s} \mathcal{D} \mathcal{W}(F) \cdot(\nabla v) F=\left.\int_{\Omega} \chi_{s} \mathcal{W}(F)\right|_{0} ^{T} .
$$

Combining the above equations results in the classical energy equation

$$
\int_{\Omega}\left[\rho\left(|v|^{2} / 2\right)+\chi_{s} \mathcal{W}(F)\right]_{0}^{T}+\int_{0}^{T} \int_{\Omega} \mu|D(v)|^{2}=\int_{\Omega} \rho f . v .
$$

Notice that in the context of a Galerkin approximation $v$ will typically be smooth, so classical solutions of the equation for $F$ can be obtained using the method of characteristics, and hence the above calculations would be justified. Upon assuming that $\sqrt{\rho} f \in L^{2}\left[0, T ; L^{2}(\Omega)\right]$ an application of the Korn and Gronwall inequalities shows that the velocity is bounded in $L^{\infty}\left[0, T ; L^{2}(\Omega)\right] \cap L^{2}\left[0, T ; H^{1}(\Omega)\right]$. This energy equation is classical $[13,14]$; the unusual treatment here being that the calculations are done in Eulerian coordinates.

2.3. Surface Tension. Balance of momentum as stated in equation (2.3) neglects surface tension. Surface tension in the fluid gives rise to a discontinuity of the normal stress, $T n$, at the solid/fluid interface proportional to the interfacial mean curvature $\kappa$. This stress is a measure supported on the surface and therefore singular; however, it is possible to approximate it using ideas of DiGorgi [6]. If $\eta$ is a smooth function then formal asymptotic expansions $[4,27,28]$ show that

$$
\lim _{\epsilon \rightarrow 0} \int_{\Omega}\left(-\epsilon \Delta \eta+(1 / \epsilon) W^{\prime}(\eta)\right) \nabla \eta . w \rightarrow \int_{\mathcal{S}}(-4 / 3) \kappa w \cdot n
$$

and

$$
\lim _{\epsilon \rightarrow 0} \int_{\Omega}\left(-\epsilon \Delta \eta+(1 / \epsilon) W^{\prime}(\eta)\right) \xi \rightarrow 0,
$$

where $W(\eta)=(1 / 2)\left(\eta^{2}-1\right)^{2}$ and $\mathcal{S}=\{x \in \Omega \mid \eta(x)=0\}$. It follows that the equations for the flow of solid/fluid systems with surface tension may be approximated by

$$
\int_{\Omega} \rho v_{t} \cdot w+\rho(v \cdot \nabla) v \cdot w+T \cdot D(w)-\gamma\left(-\epsilon \Delta \eta+(1 / \epsilon) W^{\prime}(\eta)\right) \nabla \eta \cdot w=\int_{\Omega} \rho f \cdot w
$$


with $\gamma \geq 0$ and

$$
\eta_{t}+v \cdot \nabla \eta+\gamma\left(-\epsilon \Delta \eta+(1 / \epsilon) W^{\prime}(\eta)\right)=0
$$

Notice that for incompressible solid/fluid systems the term $W^{\prime}(\eta) \nabla \eta=\nabla W(\eta)$ can be a absorbed into the pressure $p$ and that $\Delta \eta \nabla \eta=\operatorname{div}(\nabla \eta \otimes \nabla \eta)-\nabla|\nabla \eta|^{2} / 2$, and the term $\nabla|\nabla \eta|^{2} / 2$ can also be so absorbed. It is possible to identify $\eta$ with $\phi$; however, since the $T$ depends upon $\phi$ the equation for $\phi$ would require modification in order to recover an energy estimate similar to that stated in Section 2.2.

Lowengrub and Truskinovsky [24] and Gurtin, Polignone, and Vinals [15] derive equations to model the fluid/fluid problem but, use a Cahn-Hilliard equation for $\eta$ instead of the Cahn-Allen equation. This approach gives a conservation of $\eta$ and allows fluid particles to coalesce ("phase coarsening"). An integral part of the formulation of Gurtin et. al. [15] was a suitable statement of the second law of thermodynamics chosen to produce models which satisfy natural energy estimates similar to equation (2.6). The approximation of the solid/fluid problem with surface tension introduced here also satisfies a natural energy estimate; namely,

$$
\begin{aligned}
& \int_{\Omega} \rho\left(|v|^{2} / 2\right)+\chi_{s} \mathcal{W}(F)+(\epsilon / 2)|\nabla \eta|^{2}+\left.(1 / \epsilon) W(\eta)\right|_{0} ^{T} \\
& +\int_{0}^{T} \int_{\Omega} \mu|D(v)|^{2}+\gamma\left|\epsilon \Delta \eta-(1 / \epsilon) W^{\prime}(\eta)\right|^{2}=\int_{\Omega} \rho f . v .
\end{aligned}
$$

Chang et. al. [5] and more recently Li and Renardy [20] compute numerical approximations of the two fluid problem with surface tension by explicitly introducing a singular term into the momentum equation and approximating the solution of equation (2.1) using the level-set technique. In the numerical community this is considered a "competing approach" to the "phase field" ideas considered here [3, 9]. The analysis of many of these schemes is hampered by the fact that energy estimates do not hold for their particular formulations.

2.4. Deformation Gradient and Strain Energy Functions. In this section we digress slightly to discuss some technical issues associated with strain energy-functions, $\mathcal{W}$, and the structure of the evolution equation for the deformation gradient.

Strain Energy Functions. Recall that the elastic stress is zero in the fluid, so is written as $\chi_{s} \mathcal{D} \mathcal{W}(F) F$, where $\chi_{s}=(1-\phi) / 2$ is the characteristic function of the solid. This can conveniently be written as $\mathcal{D} \mathcal{W}\left(F_{s}\right) F_{s}^{T}$ with $F_{s}=\chi_{s} F$. However, this gives rise to a technical problem: physically reasonable energies are infinite when the deformation gradient (or it's determinant) vanish. Since there is no elastic stress in the fluid we are tacitly assuming that $\mathcal{D} \mathcal{W}\left(F_{s}\right) F_{s}=0$ when $F_{s}=0$.

This technical detail can be circumvented in several ways. For example, $\mathcal{W}(I)$ is finite and typically the residual stress, $\mathcal{D} \mathcal{W}(I)=0$, vanishes. We may then write the stress as

$$
\chi_{s} \mathcal{D W}(F) F^{T}=\mathcal{D} \mathcal{W}\left(F_{s}+\chi_{f} I\right) F_{s}^{T}
$$


Defining $\hat{F}=F_{s}+\chi_{f} I=\chi_{s} F+\chi_{f} I$, the elastic stress is becomes

$$
\chi_{s} \mathcal{D W}(F) F^{T}=\mathcal{D} \mathcal{W}(\hat{F})\left(\hat{F}^{T}-\chi_{f} I\right)=\mathcal{D} \mathcal{W}(\hat{F}) \hat{F}^{T}
$$

and

$$
\hat{F}_{t}+(v \cdot \nabla) \hat{F}=(\nabla v)\left(\hat{F}-\chi_{f} I\right), \quad \hat{F}(0)=I .
$$

Clearly any other stress-free state could be used in place of the identity. A variant of this approach is to write $H=F-I$ and $\overline{\mathcal{W}}(H)=\mathcal{W}(H+I)$. Then the equation for the elastic stress becomes $\mathcal{D} \overline{\mathcal{W}}(H)\left(H^{T}+\chi_{s} I\right)$ and $H$ satisfies

$$
H_{t}+(v \cdot \nabla) H=(\nabla v)\left(H+\chi_{s} I\right), \quad H(0)=0 .
$$

From a mathematical perspectives these perturbations do not change the fundamental structure of the equations, so below we will simply assume that $\mathcal{W}(0)$ is finite and write the elastic stress as $\mathcal{D} \mathcal{W}(F) F^{T}$ where $F$ satisfies equation $(2.5)$ with $F(0)=0$ in the fluid. The important structural feature is that the elastic stress takes the form $\mathcal{D} \overline{\mathcal{W}}(\bar{F}) \tilde{F}^{T}$ and $\bar{F}$ satisfies $\dot{\bar{F}}=(\nabla v) \tilde{F}$ for suitable choices of $\overline{\mathcal{W}}, \bar{F}$ and $\tilde{F}$.

Evolution Equation for the Deformation Gradient. We briefly discuss some properties of the equation for the deformation gradient. One interesting observation is that the convective derivative of the divergence of $F^{T}$, $\operatorname{div}\left(F^{T}\right)=F_{i \alpha, i}$, vanishes when $\operatorname{div}(v)=0$. To observe this, take the divergence of the equation (2.5) to obtain

$$
F_{i \alpha, i t}+v_{k} F_{i \alpha, i k}+v_{k, i} F_{i \alpha, k}=v_{i, i j} F_{j \alpha}+v_{i, j} F_{j \alpha, i} .
$$

Notice that the first term on the right vanishes since $\operatorname{div}(v)=v_{i, i}=0$, and the last term on the right is identical to the last term on the left, so that $\operatorname{div}\left(F^{T}\right)_{t}+(v \cdot \nabla) \operatorname{div}\left(F^{T}\right)=0$. It follows that $\operatorname{div}\left(F^{T}\right)$ will be zero if the initial and appropriate boundary values vanish. Unfortunately this is not so for fluid containing particles, since typically $F_{0}=\chi_{s} I$ and $\operatorname{div}\left(F^{T}\right)$ is a measure supported on the boundary of the particles. However, in the situation where $\operatorname{div}(F)=0$ the nonlinear term $(\nabla v) F$ consists of the product of a curl free term with a divergence free term, so should be stable under weak limits [30]. This becomes apparent if we consider a weak statement of equation (2.5). Letting $\Phi:(0, T) \times \Omega \rightarrow \mathbb{R}^{d \times d}$ be smooth with compact support and assuming $\operatorname{div}(v)=0$, we have

$$
\int_{0}^{T} \int_{\Omega} F \cdot\left(\Phi_{t}+(v \cdot \nabla) \Phi\right)=\int_{0}^{T} \int_{\Omega} v_{i} \Phi_{i \alpha, j} F_{j \alpha}
$$

where we used the relation $\operatorname{div}\left(F^{T}\right)=0$ to simplify the right hand side. It is now clear that granted $v_{\epsilon} \rightarrow v$ in $L^{\alpha}\left[0, T, L^{q}(\Omega)\right], \alpha, q>1$, and $F_{\epsilon} \rightarrow^{*} F$ in $L^{\infty}\left[0, T, L^{p}(\Omega)\right]$ with $1 / p+1 / q \leq 1$, then if $\left(v_{\epsilon}, F_{\epsilon}\right)$ satisfies equation $(2.7)$, then $(v, F)$ also does.

\section{Equations of a Fluid with Particles Undergoing Small Strains.}


3.1. Small Strain Elasticity. Classical linear elasticity invokes an ansatz of the form $x=X+u$ where the displacement $u$ is small $[13,14]$, so that $F=I+H$ where $H=\nabla_{X} u$. Clearly this ansatz is not plausible for elastic particles being transported in a fluid medium. Such particles will be subject to large translations and rotations, so that an ansatz of the form $x=x_{0}(t)+\hat{R}(t)(X+u)$ is plausible, where $\hat{R}$ is a rotation, and $x_{0}(t)$ is the location of the center of mass. In this situation the deformation gradient takes the form $F=\hat{R}(I+H)$. If $H$ is small, the polar decomposition is approximately $F \sim \hat{R}\left(I+H_{\text {skew }}\right)\left(I+H_{\text {sym }}\right)$. This motivates the following ansatz which we will assume throughout this section:

- The polar decomposition of the deformation gradient takes on the form $F=$ $R(I+E)$ where $R$ is a proper rotation and $E=E^{T}$ is "small".

3.2. Evolution Equations for Small Strain. We develop approximate equations satisfied by $R$ and $E$. By $\dot{F}=(\nabla v) F$ and $F=R(I+E)$,

$$
\dot{R}(I+E)+R \dot{E}=(\nabla v) R(I+E) .
$$

Pre-multiplying this equation by $R^{T}=R^{-1}$ and post multiplying by $I-E$ (an approximate inverse of $I+E$ ) gives

$$
R^{T} \dot{R}+\dot{E}=R^{T}(\nabla v) R+R^{T}(\dot{R}-(\nabla v) R) E^{2}+\dot{E} E .
$$

The latter two terms on the right of this equation are of order $O\left(E^{2}\right)$, so to first order this equation becomes

$$
R^{T} \dot{R}+\dot{E}=R^{T}(\nabla v) R .
$$

Since $R^{T} \dot{R}$ is skew we may decompose this equation into skew and symmetric components:

$$
\dot{R}=W(v) R, \quad \text { and } \quad \dot{E}=R^{T} D(v) R,
$$

where $D(v)$ and $W(v)$ are the symmetric and skew components of $\nabla v$ respectively.

3.3. Linearized Shear Relation. The elastic part of the Cauchy stress tensor is given by

$$
\begin{aligned}
\mathcal{D W}(F) F^{T} & =\mathcal{D} \mathcal{W}(R(I+E))(I+E) R^{T} \\
& =R \mathcal{D} \mathcal{W}(I+E)(I+E) R^{T} \\
& =R\left(\mathcal{D W}(I)+\mathbb{C}(E)+O\left(E^{2}\right)\right)(I+E) R^{T},
\end{aligned}
$$

where we use the notation

$$
\mathbb{C}(E)_{j \beta}=D^{2} \mathcal{W}(I)(E)_{j \beta}=\frac{\partial^{2} \mathcal{W}}{\partial F_{i \alpha} \partial F_{j \beta}}(I) E_{i \alpha}
$$

It follows that

$$
\mathcal{D W}(F) F^{T}=R\left(\mathcal{D} \mathcal{W}(I)+\mathcal{D} \mathcal{W}(I) E+\mathbb{C}(E)+O\left(E^{2}\right)\right) R^{T} .
$$

It is convenient to assume that the residual stress $\mathcal{D W}(I)$ vanishes, in which case $\mathbb{C}$ is symmetric, so to first order the Cauchy stress is given by $R \mathbb{C}(E) R^{T}$. 


\subsection{Summary of the Small Strain Problem.}

$$
\begin{gathered}
\rho(\phi)\left(v_{t}+(v \cdot \nabla) v\right)-\nabla p-\operatorname{div}\left(\mu(\phi) D(v)+R \mathbb{C}(E) R^{T}\right)=\rho(\phi) f, \\
\operatorname{div}(v)=0, \\
\phi_{t}+v \cdot \nabla \phi=0, \\
R_{t}+(v \cdot \nabla) R=W(v) R, \quad \text { and } \quad E_{t}+(v \cdot \nabla) E=R^{T} D(v) R .
\end{gathered}
$$

The initial data for $R$ is specified as $\left.R\right|_{t=0}=\chi_{s}(0) R_{0}$ where, as usual, $\chi_{s}$ is the characteristic function of the solid region. The hyperbolic nature of the evolution equation for $R$ then guarantees that $R$ vanishes in the fluid for all subsequent times.

Isotropic Elastic Stress: If the elastic stress in the solid particles is isotropic $\left(\mathbb{C}\left(Q E Q^{T}\right)=\right.$ $Q \mathbb{C}(E) Q^{T}$ for proper orthogonal $\left.Q\right)$, the equations for $R$ and $E$ can be combined to give a single equation for the elastic component of the stress. Since linear isotropic functions of symmetric matrices take the form $\mathbb{C}(E)=\alpha E+\beta \operatorname{trace}(E) I, \alpha, \beta \in \mathbb{R}$, it follows that the Cauchy stress of an isotropic incompressible material is $T_{e}=\alpha R E R^{T}$. A short calculation shows that

$$
\dot{T}_{e}-W(v) T_{e}+T_{e} W(v)=\alpha \chi_{s} D(v),
$$

which can be used in place of the equations for $R$ and $E$. This equation appears in the plasticity literature [1].

3.5. Regularization of the Rotation. It is clear that $E$ and the Cauchy stress depend nonlinearly on the rotation $R$; moreover, $R$ will not be smooth, since it satisfies a hyperbolic convection equation for which $W(v)$ enters as a coefficient. This lack of regularity is a technical obstacle to a satisfactory existence theory. To circumvent this difficulty we introduce a smooth rotation, $R_{\epsilon}$, that differs from $R$ by at most $\epsilon \sim O(E)$, which is consistent with the assumption of small strain. We begin by showing that the energy estimate is insensitive to perturbation of $R$. Then the regularity of $R_{\epsilon}$ and the energy estimate will be combined to establish existence for the small strain system.

There are many mathematical techniques for regularizing a function, the classical approach is to mollify with a smooth function of compact support. For example,

$$
R_{\epsilon_{t}}+(v . \nabla) R_{\epsilon}=W_{\epsilon}(v) R_{\epsilon},\left.\quad R_{\epsilon}\right|_{t=0}=\chi_{s} R_{0},
$$

where $W_{\epsilon}(v)=W\left(v_{\epsilon}\right)$ is the mollified spin tensor with $\epsilon>0$ fixed. A classical solution of the fluid solid problem would have $v$ Lipschitz and the particles would have regular boundaries. In this situation the particle vorticity, $\chi_{s} W$, would be of bounded variation, so that $\left\|\chi_{s}\left(W-W_{\epsilon}\right)\right\|_{L^{1}\left[0, T ; L^{1}(\Omega)\right]} \leq C \epsilon$. Granted this, a formal calculation shows that

$$
\left\|R-R_{\epsilon}\right\|_{L^{\infty}\left[0, T ; L^{1}(\Omega)\right]} \leq C\left|\chi_{s} W\right|_{L^{1}[0, T ; B V(\Omega)]} \epsilon,
$$

so that, if $\epsilon=O(E)$, such regularizations are consistent with the linear theory.

In two dimensions it is possible to explicitly write down the solution of the equation $\dot{R}=W(v) R$. This motivates a simple but elegant regularization of the rotation $R$. 
Two Dimensional Regularization. In two dimensions the spin tensor $W(v)$ may be written as $W(v)=(\omega / 2) J$, where $\omega=v_{2,1}-v_{1,2}$ is the vorticity and

$$
J=\left[\begin{array}{rr}
0 & 1 \\
-1 & 0
\end{array}\right]
$$

Then the equation for $R$ becomes $\dot{R}=(\omega / 2) J R$, which has solution ${ }^{1}$

$$
R=\exp (\Omega J) R_{0}=\left[\begin{array}{rr}
\cos (\Omega) & \sin (\Omega) \\
-\sin (\Omega) & \cos (\Omega)
\end{array}\right] R_{0},
$$

where $R_{0}$ is the initial rotation and $\Omega$ satisfies $\dot{\Omega}=\omega / 2$ with initial data $\Omega_{0}=0$. A natural regularization of $R$ is given by

$$
R_{\epsilon}=\exp \left(\Omega_{\epsilon} J\right) R_{0}=\left[\begin{array}{rr}
\cos \left(\Omega_{\epsilon}\right) & \sin \left(\Omega_{\epsilon}\right) \\
-\sin \left(\Omega_{\epsilon}\right) & \cos \left(\Omega_{\epsilon}\right)
\end{array}\right] R_{0}
$$

where $\Omega_{\epsilon}$ satisfies the regularized equation

$$
\dot{\Omega}_{\epsilon}-\epsilon^{2} \Delta \Omega=\omega / 2 .
$$

In the current context a classical solution of the solid/fluid problem $v$ would be piecewise smooth, Lipschitz continuous, and the particles would have finite perimeter. In this situation the ideas of Kruzkov [19] can be used to show that $\left\|\Omega-\Omega_{\epsilon}\right\|_{L^{\infty}\left[0, T ; L^{1}(\Omega)\right]} \leq C \epsilon$. Then

$$
R-R_{\epsilon}=\sin \left(\left(\Omega-\Omega_{\epsilon}\right) / 2\right)\left[\begin{array}{rr}
\sin \left(\left(\Omega+\Omega_{\epsilon}\right) / 2\right) & -\cos \left(\left(\Omega+\Omega_{\epsilon}\right) / 2\right) \\
\cos \left(\left(\Omega+\Omega_{\epsilon}\right) / 2\right) & \sin \left(\left(\Omega+\Omega_{\epsilon}\right) / 2\right)
\end{array}\right] R_{0},
$$

so $\left\|R-R_{\epsilon}\right\|_{L^{\infty}\left[0, T ; L^{1}(\Omega)\right]} \leq C \epsilon$. Thus if, $\epsilon=O(E)$, replacing $R$ with $R_{\epsilon}$ is consistent with our approximation of small strains.

Convection Equation. The phase variable $\phi$ appears as a coefficient in essentially every term of the momentum equation; in particular it multiplies quantities that would only converge weakly when passing to the limit in a Galerkin scheme. In order to pass to the limit it is vital to know that $\phi$ converges strongly in some $L^{p}$ space. The subtle point is that the coefficients in the equation for $\phi$ depend upon $v$ which, in the limit, has insufficient regularity to establish a classical solution. These issues were resolved by DiPerna and Lions in [8].

DiPerna and Lions introduced the concept of a "renormalized" solution. A renormalized solution is essentially a weak solution that satisfies all of the natural entropy equalities. If $\phi$ is a classical solution of $\phi_{t}+(v \cdot \nabla) \phi=0$, then so too is $\beta(\phi)$, where $\beta: \mathbb{R} \rightarrow \mathbb{R}$ is a smooth function. In the more general nonlinear situation a similar statement holds for

\footnotetext{
${ }^{1}$ Following tradition, the primitive of $\omega$ is denoted by the upper case character $\Omega$. Conflicts with the notation for the domain $\Omega \subset \mathbb{R}^{d}$ are easily resolved by context.
} 
convex functions $\beta$ and under passage to limits the equation satisfied by $\beta(\phi)$ becomes an (entropy) inequality [19].

The following theorem from [8] shows that renormalized solutions not only exist when the function $v$ is not smooth enough to establish a classical solution, but are also stable under perturbation.

Theorem 3.1 (DiPerna Lions). Let $\Omega$ be a bounded domain and suppose that:

- $\left\{v_{n}\right\}_{n=0}^{\infty} \subset L^{2}\left[0, T ; H_{0}^{1}(\Omega)\right]$ is a bounded sequence, div $\left(v_{n}(t)\right)=0$ in $\mathcal{D}^{\prime}(\Omega)$ for $t \in[0, T]$, and $v_{n} \rightarrow v$ in $L^{2}\left[0, T ; H_{0}^{1}(\Omega)\right]$;

- $\left\{\phi_{n}\right\}_{n=0}^{\infty} \subset L^{\infty}\left[0, T ; L^{\infty}(\Omega)\right]$ is a bounded sequence, satisfying

$$
\frac{\partial \phi_{n}}{\partial t}+\operatorname{div}\left(\phi_{n} v_{n}\right)=0, \quad \text { in } \mathcal{D}^{\prime}((0, T) \times \Omega),
$$

and $\phi_{n}(0) \rightarrow \phi_{0}$ in $L^{1}(\Omega)$.

Then $\left\{\phi_{n}\right\}_{n=0}^{\infty}$ converges in $C\left[0, T ; L^{p}(\Omega)\right]$, for all $1 \leq p<\infty$, to the unique renormalized solution of

$$
\frac{\partial \phi}{\partial t}+\operatorname{div}(\phi v)=0 \text { in } \mathcal{D}^{\prime}((0, T) \times \Omega),\left.\quad \phi\right|_{t=0}=\phi_{0} .
$$

In particular, if $\beta: \mathbb{R} \rightarrow \mathbb{R}$ is continuous, then $\left\{\beta\left(\phi_{n}\right)\right\}$ converges to $\beta(\phi)$ in $C\left[0, T ; L^{p}(\Omega)\right]$, $1 \leq p<\infty$, and $\beta(\phi)$ satisfies

$$
\frac{\partial \beta(\phi)}{\partial t}+\operatorname{div}(\beta(\phi) v)=0 \text { in } \mathcal{D}^{\prime}((0, T) \times \Omega),\left.\quad \beta(\phi)\right|_{t=0}=\beta\left(\phi_{0}\right),
$$

and $\int_{\Omega} \beta(\phi(T))=\int_{\Omega} \beta\left(\phi_{0}\right)$.

In Lemma 4.1 below we sketch the proof of a slight generalization of this result to systems of convection equations coupled through their right hand sides.

4. Existence for Mixtures with Linear Visco-Hyperelastic Particles. In this section we establish an existence result for the regularized small small strain theory developed above. We assume that $\Omega \subset \mathbb{R}^{d}$ is a bounded Lipschitz domain, and begin by summarizing the equations for linear visco-hyperelastic particles in a Newtonian fluid medium. Galerkin approximations of the equations

$(4.1) \int_{\Omega} \rho\left(v_{t}+v \cdot \nabla v\right) \cdot w+p \operatorname{div}(w)+\mu D(v) \cdot D(w)+R \mathbb{C}(E) R^{T} \cdot D(w)=\int_{\Omega} \rho f \cdot w$,

and

$$
\nabla \cdot v=0
$$


will be constructed with with initial data $\left.v\right|_{t=0}=v_{0} \in L^{2}(\Omega)$ satisfying $\operatorname{div}\left(v_{0}\right)=0$; boundary data $\left.v\right|_{\partial \Omega}=0$; and non-homogeneous term $f \in L^{2}\left[0, T ; L^{2}(\Omega)\right]$. The phase function and strain will be solutions of the equations

$$
\phi_{t}+v \cdot \nabla \phi=0
$$

and

$$
E_{t}+(v \cdot \nabla) E=R^{T} D(v) R .
$$

The density and viscosity are then determined by

$$
\rho=\chi_{f} \rho_{f}+\chi_{s} \rho_{s}, \quad \mu=\chi_{f} \mu_{f}+\chi_{s} \mu_{s}
$$

with $\chi_{f}=(1+\phi) / 2$ and $\chi_{s}=(1-\phi) / 2$.

To compute the rotation matrix fix $\epsilon>0$ and let $R$ satisfy

$$
R_{t}+(v \cdot \nabla) R=W_{\epsilon}(v) R,\left.\quad R\right|_{t=0}=\chi_{s} R_{0},
$$

where $W_{\epsilon}(v)=W\left(v_{\epsilon}\right)$ is the mollified spin tensor.

Alternatively, in two dimensions compute

$$
\Omega_{t}+v \cdot \nabla \Omega-\epsilon^{2} \Delta \Omega=\operatorname{curl}(v),\left.\quad \Omega\right|_{t=0}=0, \quad \partial \Omega / \partial n=0,
$$

and set

$$
R=\chi_{s}\left[\begin{array}{rr}
\cos (\Omega) & \sin (\Omega) \\
-\sin (\Omega) & \cos (\Omega)
\end{array}\right] R_{0}
$$

Solutions will be obtained as limits of Galerkin approximations and will satisfy:

$$
v \in L^{\infty}\left[0, T ; L^{2}(\Omega)\right] \cap L^{2}\left[0, T ; H_{0}^{1}(\Omega)\right], \quad \phi, R \in L^{\infty}\left[0, T ; L^{\infty}(\Omega)\right], \quad E \in L^{\infty}\left[0, T ; L^{2}(\Omega)\right] ;
$$

$\operatorname{div}(v)=0$, and a weak form of the momentum equation, namely

$\int_{0}^{T} \int_{\Omega}-\rho v \cdot w_{t}-(\rho v \otimes v) \cdot \nabla w+\mu D(v) \cdot D(w)+\mathbb{C}(E) \cdot R^{T} D(w) R=\int_{\Omega} \rho_{0} v_{0} \cdot w(0)+\int_{0}^{T} \int_{\Omega} \rho f \cdot w$

for all $w \in \mathcal{D}([0, T) \times \Omega)$ with $\operatorname{div}(w)=0$. The equations for $\phi, E$ and $R$ (and, if applicable, the vorticity $\Omega$ ) will be satisfied in the usual weak sense.

4.1. Estimates for the Small Strain System. There are two important structural differences between these equations and the complete system (2.2)-(2.5). While they both satisfy an energy estimate, the elastic stress in the above system will be in $L^{2}$ instead of $L^{1}$, and, unlike equation (2.5) for the deformation gradient, the equation for the linearized strain will directly give estimates for $E$. However, one important feature is lost; namely the term $W(v) R$ in the equation for $R$, while very similar to the corresponding term $(\nabla v) F$ in the equation for the deformation gradient, does not 
have the div-curl structure; this is what forces the introduction of the regularizations discussed above.

The derivation of the energy estimate for the system with linearized elastic stress is obtained by selecting $w=v$ in the weak statement of the momentum equation, taking the (Frobenius) inner product of the equation for $E$ with $\mathbb{C}(E)$, and adding the resulting equations. As in the original equations, balance of mass and integration by parts enable the sum of the kinetic and elastic energies to be estimated by

$$
\begin{aligned}
\frac{1}{2} \int_{\Omega}\left(\rho(T)|v(T)|^{2}+|E(T)|_{\mathbb{C}}^{2}\right) & +\int_{0}^{T} \int_{\Omega} \mu|D(v)|^{2} \\
& =\frac{1}{2} \int_{\Omega}\left(\rho_{0}\left|v_{0}\right|^{2}+\left|E_{0}\right|_{\mathbb{C}}^{2}\right)+\int_{0}^{T} \int_{\Omega} \rho f . v .
\end{aligned}
$$

These calculations require some regularity on $v$; the Galerkin approximation will only assume this equation to hold for smooth velocities.

As stated above, one of the major differences between the evolution equations for the deformation gradient $F$ and its linearized counterpart is that the later directly yields bounds. In particular,

$$
\frac{1}{2} \frac{d}{d t} \int_{\Omega}|E|^{2} \leq \int_{\Omega}|D(v)||E|
$$

so

$$
\|E(T)\|_{L^{2}(\Omega)} \leq\|E(0)\|_{L^{2}(\Omega)}+\int_{0}^{T}\|D(v)\|_{L^{2}(\Omega)} .
$$

Finally, it is necessary to establish the stability of solutions of the equation for $R$ under perturbations of the velocity. Given a sequence of velocity fields $\left\{v_{n}\right\}_{n=0}^{\infty}$ converging weakly in $L^{2}\left[0, T ; H_{0}^{1}(\Omega)\right]$, their spins $\left\{W\left(v_{n}\right)\right\}$ will converge weakly in $L^{2}\left[0, T ; L^{2}(\Omega)\right]$. If $\left\{W_{\epsilon}\left(v_{n}\right)\right\}$ are the mollified spin tensors and $\Phi \in \mathcal{D}((0, T) \times \Omega)$, then $W_{\epsilon}\left(v_{n}\right) \Phi$ will converge weakly in $L^{2}\left[0, T ; H_{0}^{1}(\Omega)\right]$; indeed, if $\Psi$ is smooth,

$$
\begin{aligned}
\int_{0}^{T} \int_{\Omega} \nabla\left(W_{\epsilon}\left(v_{n}\right) \Phi\right) \cdot \nabla \Psi & =\int_{0}^{T} \int_{\Omega}-W_{\epsilon}\left(v_{n}\right) \Phi \cdot \Delta \Psi \\
& =\int_{0}^{T} \int_{\Omega}-W_{\epsilon}\left(v_{n}\right) \cdot(\Delta \Psi) \Phi^{T} \\
& =\int_{0}^{T} \int_{\Omega}-W\left(v_{n}\right) \cdot\left((\Delta \Psi) \Phi^{T}\right)_{\epsilon} \\
& \rightarrow \int_{0}^{T} \int_{\Omega}-W(v) \cdot\left((\Delta \Psi) \Phi^{T}\right)_{\epsilon} \\
& =\int_{0}^{T} \int_{\Omega} \nabla\left(W_{\epsilon}(v) \Phi\right) \cdot \nabla \Psi
\end{aligned}
$$

In this situation the following lemma shows that the sequence of rotations computed from $\left\{W\left(v_{\epsilon}\right)\right\}$ will converge strongly. 
LEMMA 4.1. Let $\left\{v_{n}\right\}_{n=1}^{\infty}$ be a sequence of smooth functions that converge weakly in $L^{2}\left[0, T ; H_{0}^{1}(\Omega)\right]$ and satisfy div $\left(v_{n}\right)=0$, and let $\left\{W_{n}\right\}_{n=0}^{\infty}$ be a sequence of smooth skew matrices bounded in $L^{2}\left[0, T ; L^{2}(\Omega)\right]$. Suppose that $W_{n} \Phi \rightarrow W \Phi$ in $L^{2}\left[0, T ; H_{0}^{1}(\Omega)\right]$ for every smooth test function $\Phi \in \mathcal{D}((0, T) \times \Omega)$ and that $R_{n}$ satisfies

$$
R_{n_{t}}+v_{n} . \nabla R_{n}=W_{n} R_{n},\left.\quad R_{n}\right|_{t=0}=R_{n 0} \in L^{\infty}(\Omega) \cap L^{2}(\Omega),
$$

where the initial data $\left\{R_{n 0}\right\}$ to $R_{0}$ converge in $L^{2}(\Omega)$. The sequence $\left\{R_{n}\right\}$ is then bounded in $L^{\infty}\left[0, T ; L^{\infty}(\Omega)\right] \cap L^{\infty}\left[0, T ; L^{2}(\Omega)\right]$ and converges in $L^{2}\left[0, T ; L^{2}(\Omega)\right]$ (and hence all $\left.L^{p}\left[0, T ; L^{p}(\Omega)\right], 1 \leq p<\infty\right)$ to a weak solution of

$$
R_{t}+v \cdot \nabla R=W R,\left.\quad R\right|_{t=0}=R_{0} .
$$

Proof. The proof of strong convergence is a mild generalization of the results of DiPerna and Lions [8]; the major difference is that in the scalar case it is necessary for $\left\{W_{n}\right\}$ to be bounded in $L^{\infty}$ while for the coupled system of equations the assumption that $W \in$ $L^{2}\left[0, T ; L^{2}(\Omega)\right]$ and skew suffices. The idea of the proof is quite elementary; however, one step requires a technical result from [8] or [22] to justify a formal calculation.

The $L^{\infty}$ bound on $\left\{R_{n}\right\}$ is immediate. Writing the equation as $R_{n}^{T} \dot{R}_{n}=R_{n}^{T} W_{n} R_{n}$ and adding this to it's transpose gives

$$
\left(R_{n}^{T} R_{n}\right)^{\cdot}=R_{n}^{T}\left(W_{n}+W_{n}^{T}\right) R_{n}=0,\left.\quad R_{n}^{T} R_{n}\right|_{t=0}=R_{n 0}^{T} R_{n 0} .
$$

Since $|R|=\operatorname{trace}\left(R^{T} R\right)$ the $L^{\infty}$ bound follows. Similarly, since $\left(\left|R_{n}\right|^{2} / 2\right)=\dot{R}_{n} \cdot R_{n}=$ $W_{n} R_{n} \cdot R_{n}=0$ it follows that $\left\|R_{n}(t)\right\|_{L^{2}(\Omega)}=\left\|R_{n 0}\right\|_{L^{2}(\Omega)}$, and we explicitly compute $\left\|R_{n}\right\|_{L^{2}\left[0, T ; L^{2}(\Omega)\right]}=\sqrt{T}\left\|R_{n 0}\right\|_{L^{2}(\Omega)}$.

The bounds show that we may pass to a subsequence $\left\{R_{n}\right\}$ which converges weakly in $L^{2}\left[0, T ; L^{2}(\Omega)\right]$ to a limit $R \in L^{\infty}\left[0, T ; L^{\infty}(\Omega)\right] \cap L^{\infty}\left[0, T ; L^{2}(\Omega)\right]$. Integration by parts shows that

$$
\int_{0}^{T} \int_{\Omega} R_{n, t} \cdot \Phi=\int_{0}^{T} \int_{\Omega} W_{n} R_{n} \cdot \Phi+R_{n} \cdot\left(v_{n} \cdot \nabla\right) \Phi
$$

for any smooth function, hence $\left\{R_{n, t}\right\}$ is bounded in $L^{2}\left[0, T ; H^{-1}(\Omega)\right]$. The LionsAubin lemma [31] then shows that, upon passing to a subsequence, $R_{n} \rightarrow R$ strongly in $C\left[0, T ; H^{-1}(\Omega)\right]$. The hypotheses on the coefficients $v_{n}$ and $W_{n}$ then suffice to pass to the limit term by term in the weak statement

$$
\int_{0}^{T} \int_{\Omega} R_{n} \cdot\left(\Phi_{t}+\left(v_{n} . \nabla\right) \Phi-W_{n} \Phi\right)=\left.\int_{\Omega} R_{n 0} \cdot \Phi\right|_{t=0},
$$

so that $R$ is a weak solution of $R_{t}+v \cdot \nabla R=W R$ with initial data $R_{0}$. At this point we would like to take the dot product of this equation with $R$ to conclude $\dot{R} \cdot R=0$ and hence $\|R\|_{L^{2}\left[0, T ; L^{2}(\Omega)\right]}=\sqrt{T}\left\|R_{0}\right\|_{L^{2}(\Omega)}$. However, such a computation would be formal since the weak solutions are not sufficiently smooth to carry out this computation. 
To circumvent this technical problem DiPerna and Lions [8] considered the equation satisfied by mollifications $R_{\eta}$ of $R . R_{\eta}$ satisfies

$$
R_{\eta_{t}}+v \cdot \nabla R_{\eta}=W R_{\eta}+O(\eta)
$$

where $O(\eta)$ is an error term which, under the regularity hypotheses assumed for $v$ and $W$, converges to zero in $L^{1}\left[0, T ; L^{1}(\Omega)\right]+L^{2}\left[0, T ; L^{2}(\Omega)\right]$ as $\eta \rightarrow 0$. Taking the inner product with $R_{\eta}$ gives

$$
\left\|R_{\eta}\right\|_{L^{2}\left[0, T ; L^{2}(\Omega)\right]}=\sqrt{T}\left\|R_{0}\right\|_{L^{2}(\Omega)}+2 \int_{0}^{T} \int_{\Omega} O(\eta) \cdot R_{\eta},
$$

and passing to the limit $\eta \rightarrow 0$ (and recalling that $R$ is bounded in $L^{\infty} \cap L^{2}$ ) we obtain

$$
\|R\|_{L^{2}\left[0, T ; L^{2}(\Omega)\right]}=\sqrt{T}\left\|R_{0}\right\|_{L^{2}(\Omega)}=\lim _{n \rightarrow \infty} \sqrt{T}\left\|R_{n 0}\right\|_{L^{2}(\Omega)}=\lim _{n \rightarrow \infty}\left\|R_{n}\right\|_{L^{2}\left[0, T ; L^{2}(\Omega)\right]},
$$

so that the weak convergence of $\left\{R_{n}\right\}$ is actually strong. Notice that the mollification argument shows that weak solutions are unique since the difference of two weak solutions is a weak solution with zero initial data. We then conclude that the whole sequence $\left\{R_{n}\right\}$ converges strongly to $R$.

Next, consider the two dimensional situation where $R$ is computed using equation (4.5). If $\operatorname{div}(v)=0$, the natural estimate for $\Omega$ is

$$
\frac{1}{2} \frac{d}{d t} \int_{\Omega}|\Omega|^{2}+\epsilon^{2} \int_{\Omega}|\nabla \Omega|^{2}=\int_{\Omega} \omega \Omega,
$$

where $\omega=\operatorname{curl}(v)=v_{2,1}-v_{1,2} \in L^{2}(\Omega)$. A Gronwall argument then shows that

$$
\|\Omega(T)\|_{L^{2}(\Omega)}^{2}+2 \epsilon^{2} \int_{0}^{T}\|\nabla \Omega\|_{L^{2}(\Omega)}^{2} \leq e^{T} \int_{0}^{T}\|\omega\|_{L^{2}(\Omega)}^{2}
$$

(recall that $\Omega(0)=0$ ). It follows that a bound upon the velocity in $L^{2}\left[0, T ; H^{1}(\Omega)\right]$ gives bounds upon $\Omega$ in $C\left[0, T ; L^{2}(\Omega)\right] \cap L^{2}\left[0, T ; H^{1}(\Omega)\right]$. Since $\Omega_{t}$ is bounded in $L^{2}\left[0, T ; H^{-1}(\Omega)\right]$, the Lions Aubin lemma [31] shows that the mapping $v \mapsto \Omega$ is "completely continuous" (compact) from $L^{2}\left[0, T ; H^{1}(\Omega)\right]$ into $L^{2}\left[0, T ; L^{2}(\Omega)\right]$.

4.2. Existence of Solutions. To establish existence of solutions to equations (4.1)-(4.3) (or (4.5)) we utilize a Galerkin scheme. Let $V_{1} \subset V_{2} \subset \cdots \subset H_{0}^{1}(\Omega)$ be a sequence of finite dimensional spaces of smooth divergence free functions, and let $\cup_{n} V_{n}$ be dense in $V=\left\{v \in H_{0}^{1}(\Omega) \mid \operatorname{div}(v)=0\right\}$. For definiteness let $V_{n}$ be spanned by a sequence $\left\{w_{j}\right\}_{j=1}^{n}$, where $\left\{w_{j}\right\}_{j=1}^{\infty}$ is a dense set of $V$. For $v \in V_{n}$, define $(\phi(v), R(v), E(v))$ to be the solutions of equations (2.1), (4.3), and (4.2) respectively, with coefficients determined by $v$. Since functions in $V_{n}$ are smooth, classical solutions of these equations can be computed using the method of characteristics. It is then possible to construct a map $\mathcal{F}: C\left[0, T, V_{n}\right] \rightarrow C\left[0, T, V_{n}\right]$ by defining $v=\mathcal{F}(\hat{v})$ to be the approximate solution 
of equation (4.1) with coefficients $(\phi(\hat{v}), R(\hat{v}), E(\hat{v}))$ obtained by restricting the solution and test functions to be in $V_{n}$.

The following lemma shows that the mapping $\mathcal{F}$ is not only well defined but has a fixed point. The bounds derived from the energy estimate will then suffice to show that a subsequence of fixed points, $\left\{v_{n}\right\}$, converge to a limit satisfying equations (4.1)-(4.3) (or $(4.5))$. If the initial data $v_{0}$ is not smooth, select the initial value for the approximate problem to be the $H^{1}$ projection of $v_{0}$ into $V_{n}$.

LEMMA 4.2. Let $V_{n}$ be a finite dimensional space of smooth divergence-fee functions $\left(\operatorname{div}(v)=0\right.$ for $\left.v \in V_{n}\right)$.

1. The mapping $\mathcal{F}: C\left[0, T, V_{n}\right] \rightarrow C\left[0, T ; V_{n}\right]$, defined above, exists for sufficiently small times $T>0$.

2. For each $T>0, \mathcal{F}$ has a fixed point $v_{n} \in C\left[0, T ; V_{n}\right]$, and $v_{n}$ satisfies the energy estimate (4.6).

Proof. Step 1: For $\hat{v} \in V_{n}$, classical techniques can be used to compute the coefficients $(\phi(\hat{v}), R(\hat{v}), E(\hat{v}))$, and it is clear that their integrals vary continuously with respect to time. In this situation the Galerkin approximation of equation (4.1) reduces to a system of first order ordinary differential equations in $t$ where the "right hand side" is a locally Lipschitz function. Piccard's theorem then establishes existence of a solution $v$ for small times. Next, substitute $w=v-v_{0}$ into equation (4.1) to obtain

$$
\begin{aligned}
\int_{\Omega} \hat{\rho}\left(\left|v-v_{0}\right|^{2} / 2\right)_{t}+(\hat{\rho} \hat{v}) \cdot \nabla\left(\left|v-v_{0}\right|^{2} / 2\right) & +\hat{\mu}\left|D\left(v-v_{0}\right)\right|^{2}+\hat{R}^{T} \mathbb{C}(\hat{E}) \hat{R} \cdot D\left(v-v_{0}\right) \\
& =\int_{\Omega} \hat{\rho} f \cdot\left(v-v_{0}\right)-\hat{\mu} D\left(v_{0}\right) \cdot D\left(v-v_{0}\right),
\end{aligned}
$$

where we have written $\hat{\phi}=\phi(\hat{v})$ etc. Since $\hat{\rho}_{t}+\nabla \cdot(\hat{v} \hat{\rho})=0$, it follows that

$$
\int_{\Omega} \hat{\rho}(T)\left|v(T)-v_{0}\right|^{2} \leq C\left(\|f\|_{L^{2}\left[0, T ; L^{2}(\Omega)\right]},\left\|v_{0}\right\|_{H^{1}(\Omega)}\right) \int_{0}^{T}\left(1+\|\hat{v}\|_{H^{1}(\Omega)}^{2}\right) .
$$

To obtain this estimate we used the fact that $\hat{\phi}, \hat{R} \in L^{\infty}$, and hence so too are $\hat{\mu}$ and $\hat{\rho}$, and equation (4.7) was used to bound $\hat{E}$. Since $\hat{\rho} \geq \min \left(\rho_{f}, \rho_{s}\right)>0$, and since all norms on finite dimensional spaces are equivalent, it follows that

$$
\left\|v-v_{0}\right\|_{C\left[0, T ; V_{n}\right]}^{2} \leq C_{n} T\left(1+\left\|\hat{v}-v_{0}\right\|_{C\left[0, T ; V_{n}\right]}^{2}\right),
$$

so if $T \leq 1 / 2 C_{n}$, the function $v=\mathcal{F}(\hat{v})$ maps a ball in $C\left[0, T, V_{n}\right]$ centered at $v_{0}$ into itself.

Step 2: In the above we tacitly assumed that $v_{0}$ was the initial data; however, if $v_{0}=v\left(t_{0}\right)$ for some $0 \leq t_{0} \leq T$, then the above estimate shows that

$$
\left\|v(t)-v\left(t_{0}\right)\right\|_{V_{n}}^{2} \leq C_{n}\left(v_{0}, \hat{v}\right)\left|t-t_{0}\right|
$$


and hence $v \in C\left[0, T, V_{n}\right]$ is Lipschitz. It then follows from the Arzela-Ascoli theorem that $\mathcal{F}$ is a "completely continuous" (compact) mapping from the unit ball in $C\left[0, T, V_{n}\right]$ centered at $v_{0}$ to itself. The Schauder fixed point theorem then establishes the existence of a fixed point, $v_{n}=\mathcal{F}\left(v_{n}\right)$.

The energy estimate (4.6) now yields

$\int_{\Omega}\left(\rho_{n}\left|v_{n}(T)\right|^{2} / 2+\left|E_{n}(T)\right|_{\mathbb{C}}^{2}\right)+\int_{0}^{T} \int_{\Omega} \mu_{n}\left|D\left(v_{n}\right)\right|^{2}=\int_{\Omega}\left(\rho_{0}\left|v_{0}\right|^{2} / 2+\left|E_{0}\right|_{\mathbb{C}}^{2}\right)+\int_{0}^{T} \int_{\Omega} \rho_{n} f \cdot v_{n}$.

It follows that the fixed point $v_{n}$ is uniformly bounded in time, and the above argument, which guaranteed solutions for short times, can be repeated indefinitely to obtain existence of a solution in $C\left[0, T ; V_{n}\right]$ satisfying the energy estimates for arbitrarily large T.

To verify that the sequence of Galerkin approximations converge we will need the following compactness result of J. L. Lions [21]. This theorem was developed by Lions to establish existence results for incompressible fluids with non-constant density and has been used frequently in this context [23].

THEOREM 4.3 (J. L. Lions). Let $\Omega \subset \mathbb{R}^{3}$ be a bounded domain and suppose the sequence $\left\{v_{n}\right\}_{n=1}^{\infty}$ is bounded in $L^{\infty}\left[0, T ; L^{2}(\Omega)\right] \cap L^{2}\left[0, T ; H_{0}^{1}(\Omega)\right]$, and that there exists $C$ and $\alpha>0$ such that, for all $0 \leq \delta<1$,

$$
\int_{0}^{T-\delta}\left|v_{n}(t+\delta)-v_{n}(t)\right|^{2} \leq C \delta^{\alpha}, \quad n=1,2, \ldots
$$

Then the sequence is relatively compact in $L^{p}\left[0, T ; L^{q}(\Omega)\right]$ for any pair $(p, q)$ satisfying $2 / p+3 / q>3 / 2$.

This theorem follows from a classical result of Frechet and Kolmogorov, see [29, page $50]$, which is a variant of the Arzela-Ascoli theorem applicable to $L^{p}(\Omega)$ spaces.

THEOREM 4.4. Equations (4.1)-(4.3) (or (4.5)) with the assumptions on the boundary and initial data stated at the beginning of this section have a weak solution satisfying the energy estimate (4.6) (with inequality).

Proof. Let $\left\{v_{n}, \phi_{n}, E_{n}, R_{n}\right\}_{n=0}^{\infty}$ be the Galerkin approximations constructed in the lemma. The lower bound $\rho_{n} \geq \min \left(\rho_{f}, \rho_{s}\right)$ and the energy estimate directly yield bounds upon $v_{n}$ in $L^{\infty}\left[0, T ; L^{2}(\Omega)\right] \cap L^{2}\left[0, T ; H^{1}(\Omega)\right]$, and by construction $\operatorname{div}\left(v_{n}\right)=0$. The hypotheses of Theorem 3.1 are then satisfied by the sequence $\left\{\left(v_{n}, \phi_{n}\right)\right\}$ and, upon passing to a subsequence, we conclude that there exists $\phi \in L^{\infty}\left[0, T ; L^{\infty}(\Omega)\right]$ such that $\phi_{n} \rightarrow \phi$ in $C\left[0, T ; L^{p}(\Omega)\right]$ for all $1 \leq p<\infty$. Since $\rho_{n}, \mu_{n}$ etc. are all affine functions of $\phi_{n}$ these quantities converge similarly.

We utilize the technique of J. L. Lions [21] to establish strong convergence of the velocities in $L^{2}\left[0, T ; L^{2}(\Omega)\right]$. The densities $\left\{\rho_{n}\right\}$ each satisfy equation $(2.2)$, so if $0 \leq$ 
$\delta<1$ and $\psi \in H_{0}^{1}(\Omega)$ it follows that

$$
\int_{\Omega}\left(\rho_{n}(t+\delta)-\rho_{n}(t)\right) \psi=\int_{t}^{t+\delta} \int_{\Omega} \rho_{n} v_{n} . \nabla \psi .
$$

Putting $\psi=v_{n}(t) . w$ into this equation gives

$$
\int_{\Omega}\left(\rho_{n}(t+\delta)-\rho(t)\right) v_{n}(t) \cdot w=\int_{t}^{t+\delta} \int_{\Omega} \rho_{n}(s) v_{n}(s) \cdot \nabla\left(v_{n}(t) \cdot w\right) d s .
$$

Next, if $w \in V_{n}$, equation (4.1) and equation (2.2) may be combined to yield

$$
\begin{aligned}
& \int_{\Omega}\left(\rho_{n}(t+\delta) v_{n}(t+\delta)-\rho_{n}(t) v_{n}(t)\right) \cdot w \\
& =\int_{t}^{t+\delta} \int_{\Omega}\left(\rho v_{n} \otimes v_{n}\right) \cdot \nabla w-\mu D\left(v_{n}\right) \cdot D(w)-R \mathbb{C}\left(E_{n}\right) R^{T} \cdot D(w) .
\end{aligned}
$$

Subtracting the previous two equations gives

$$
\begin{aligned}
& \int_{\Omega} \rho_{n}(t+\delta)\left(v_{n}(t+\delta)-v_{n}(t)\right) \cdot w \\
& =\int_{t}^{t+\delta} \int_{\Omega}\left(\rho v_{n} \otimes v_{n}\right) \cdot \nabla w-\mu D\left(v_{n}\right) \cdot D(w)-R \mathbb{C}\left(E_{n}\right) R^{T} \cdot D(w)-\rho_{n} v_{n} \cdot \nabla\left(v_{n}(t) \cdot w\right),
\end{aligned}
$$

where we have suppressed the variable of integration on the right.

Recalling that $R \in L^{\infty}$ and $\mathbb{C}: \mathbb{R}^{d \times d} \rightarrow \mathbb{R}^{d \times d}$ is a bounded linear map, it follows that

$$
\begin{aligned}
& \left|\int_{\Omega} \rho_{n}(t+\delta)\left(v_{n}(t+\delta)-v_{n}(t)\right) . w\right| \leq \int_{t}^{t+\delta}\left\|v_{n}\right\|_{L^{4}(\Omega)}\left\|\nabla v_{n}(t)\right\|_{L^{2}(\Omega)}\|w\|_{L^{4}(\Omega)}+ \\
& C \int_{t}^{t+\delta}\left(\left\|v_{n}\right\|_{L^{4}(\Omega)}^{2}+\left\|D\left(v_{n}\right)\right\|_{L^{2}(\Omega)}+\left\|E_{n}\right\|_{L^{2}(\Omega)}+\left\|v_{n}\right\|_{L^{4}(\Omega)}\left\|v_{n}(t)\right\|_{L^{4}(\Omega)}\right)\|\nabla w\|_{L^{2}(\Omega)} .
\end{aligned}
$$

The Sobolev embedding theorem states that $\|v\|_{L^{4}(\Omega)} \leq C\|v\|_{L^{2}(\Omega)}^{1-\alpha}\|v\|_{H^{1}(\Omega)}^{\alpha}$ where $\alpha=$ $1 / 2$ in two dimensions and $\alpha=3 / 4$ in three dimensions. Since $v_{n}$ is bounded in $L^{\infty}\left[0, T ; L^{2}(\Omega)\right]$, the bounds from the energy estimate and the bound (4.7) show that the integrands on the right are in $L^{1 / \alpha}$, then

$$
\begin{aligned}
& \left|\int_{\Omega} \rho_{n}(t+\delta)\left(v_{n}(t+\delta)-v_{n}(t)\right) \cdot w\right| \\
& \leq C\left(\left\|\nabla v_{n}(t)\right\|_{L^{2}(\Omega)}\|w\|_{L^{4}(\Omega)}+\left(1+\left\|v_{n}(t)\right\|_{L^{4}(\Omega)}\right)\|\nabla w\|_{L^{2}(\Omega)}\right) \delta^{1-\alpha} \\
& \leq C\left(\left\|\nabla v_{n}(t)\right\|_{L^{2}(\Omega)}\|w\|_{L^{4}(\Omega)}+\|\nabla w\|_{L^{2}(\Omega)}+\left\|\nabla v_{n}(t)\right\|_{L^{2}(\Omega)}^{\alpha}\|\nabla w\|_{L^{2}(\Omega)}\right) \delta^{1-\alpha} .
\end{aligned}
$$

Finally, put $w=v_{n}(t+\delta)-v_{n}(t)$ and verify that the right hand side is integrable to obtain

$\min \left(\rho_{f}, \rho_{s}\right) \int_{0}^{T-\delta}\left\|v_{n}(t+\delta)-v_{n}(t)\right\|_{L^{2}(\Omega)} \leq \int_{0}^{T-\delta} \int_{\Omega} \rho_{n}(t+\delta)\left|v_{n}(t+\delta)-v_{n}(t)\right|^{2} \leq C \delta^{1-\alpha}$. 
This verifies the final hypothesis of Theorem 4.3, so we conclude that $\left\{v_{n}\right\}$ is relatively compact in $L^{2}\left[0, T ; L^{2}(\Omega)\right]$, and may pass to a subsequence for which $v_{n} \rightarrow v$ strongly in $L^{2}\left[0, T ; L^{2}(\Omega)\right]$.

The bounds upon $v_{n}$ provided by the energy estimate and the strong convergence of (a subsequence of) $\left\{v_{n}\right\}$ establish the hypotheses for Lemma (4.1), so that the subsequence $\left\{R_{n}\right\}$ of (regularized) rotations converge strongly in $L^{p}\left[0, T ; L^{p}(\Omega)\right], 1 \leq p<\infty$. In two dimensions the strong convergence of the sequence $\Omega_{n}$ and the identity (3.1) lead to the same conclusion. Finally, since $E_{n}$ is bounded in $L^{\infty}\left[0, T ; L^{2}(\Omega)\right]$ is is possible to pass to a subsequence which converges weakly star in this space.

We are now in a position to show that the limit $(v, \phi, E, R)$ of a subsequence of Galerkin approximations is a weak solution of equations (4.1)-(4.3) (or (4.5)). Let $w \in \mathcal{D}([0, T) \times$ $\Omega$ ) satisfy $\operatorname{div}(w)=0$, then by density there exists a sub-sequence $\left\{\hat{w}_{n}\right\}_{n=0}^{\infty}$ with $\hat{w}_{n} \in$ $C^{1}\left[0, T ; V_{n}\right]$ such that $\hat{w}_{n} \rightarrow w$ in $C^{1}\left[0, T ; W^{1, q}(\Omega)\right]$ for $q \geq 1$. Since it is possible to select $\hat{w}_{n}(T)=0$, each Galerkin approximation satisfies

$$
\begin{aligned}
\int_{0}^{T} \int_{\Omega}-\rho_{n} v_{n} \cdot \hat{w}_{n_{t}}-\left(\rho_{n} v_{n} \otimes v_{n}\right) \cdot \nabla \hat{w}_{n} & +\mu_{n} D\left(v_{n}\right) \cdot D\left(\hat{w}_{n}\right)+\mathbb{C}\left(E_{n}\right) \cdot R_{n}^{T} D\left(\hat{w}_{n}\right) R_{n} \\
& =\int_{\Omega} \rho_{n}(0) v_{n}(0) \cdot \hat{w}_{n}(0)+\int_{0}^{T} \int_{\Omega} \rho_{n} f \cdot \hat{w} .
\end{aligned}
$$

The first two terms in this equation are the product of functions which converge in $L^{p}\left[0, T ; L^{p}(\Omega)\right], p>1$, and gradients of the test function which converges strongly in $L^{p^{\prime}}\left[0, T ; L^{p^{\prime}}(\Omega)\right]$. It follows that these terms converge strongly and hence pass to their natural limits. This argument also shows that the terms $\mu_{n} D\left(\hat{w}_{n}\right)$ and $R_{n}^{T} D\left(\hat{w}_{n}\right) R_{n}$ converge strongly in $L^{2}\left[0, T ; L^{2}(\Omega)\right]$, and since $D\left(v_{n}\right)$ and $E_{n}$ converge weakly it is again possible to pass to the limit. It follows that

$\int_{0}^{T} \int_{\Omega}-\rho v \cdot w_{t}-\rho(v \otimes v) \cdot \nabla w+\mu D(v) \cdot D(w)+\mathbb{C}(E) \cdot R^{T} D(w) R=\int_{\Omega} \rho_{0} v_{0} \cdot w(0)+\int_{0}^{T} \int_{\Omega} \rho f \cdot w$

for all $w \in \mathcal{D}([0, T) \times \Omega)$ satisfying $\operatorname{div}(w)=0$. This line of argument is equally applicable to weak statements of equations (4.2) and equation (4.3) (or (4.4)) and shows that they are satisfied by the limits $E$ and $R$ (and $\Omega$ ).

\section{REFERENCES}

[1] L. Anand And M. Kothari, A computations procedure for rate independent cyrstal placticity, J. Mech. Phys. Solids, 4 (1996), pp. 525-558.

[2] R. J. Asaro, Crystal placticity, J. of Applied Mechancis, 50 (1983), pp. 921-934.

[3] G. Caginalp, An analysis of a phase field model of a free boundary, Archive for Rational Mechanics and Analysis, 92 (1986), pp. 205-245. 
[4] - Stefan and Hele-Shaw type models a asymptotic limits of phase field equations, Physics Review A, 39 (1989), pp. 887-896.

[5] Y. C. Chang, T. Y. Hou, B. Merriman, and S. Osher, A level set formulation of Eulerian interface capturing methods for incompressible fluid flows., J. Comput. Phys., 124 (1996), pp. $449-464$.

[6] E. DeGiongi, Some conjectures on flow by mean curvature, in Methods of Real analysis and Partial Differential Equations, M. L. Benevento, T. Bruno, and C. Sbordone, eds., Liguori, Napoli, 1990.

[7] S. Demoulini, Weak solutions for a class of nonlinear systems of viscoelasticity, Archive for Rational Mechanics and Analysis, Submitted (2000).

[8] R. J. DiPerna and P. L. Lions, Ordinary differential equations, transport theory and Sobolev spaces, Inventiones Mathematicae, 98 (1989), pp. 511-547.

[9] G. Fix, Phase field methods for free boundary problems, in Free Boundary Problems, B. Fasano and M. Primicerio, eds., Pitman, London, 1983, pp. 580-589.

[10] E. Freid And M. E. Gurtin, Continuum theory of thermally induced phase transitions based on an order parameter, Physica D, 68 (1993), pp. 326-343.

[11] J. Glimm, J. Grove, , B. Plohr, D. Sharp, and F. Wang, A conservative Eulerian numerical scheme for elastoplasticity and application to plate impact problems, Impact Comput. Sci. Engrg., 5 (1993), pp. 285-308.

[12] R. Glowinski, T. Pan, T. Hesla, D. Joseph, and J. Periaux, A distributed Lagrange multiplier/fictitious domain method for the simulation of flow around moving rigid bodies: application to particulate flow, Comput. Methods Appl. Mech. Engrg., 184 (2000), pp. 241267.

[13] M. E. GurTin, Linear Theories of Elasticity and Thermoelasticity, no. II in Mechanics of Solids, Springer-Verlag, 1973.

[14] — An introduction to Continuum Mechanics, no. 158 in Mathematics in Science and Engineering, Academic Press, 1981.

[15] M. E. Gurtin, D. Polignone, And J. Viñals, Two-phase binary fluids and immiscible fluids described by an order parameter, Math. Models Methods Appl. Sci., 6 (1996), pp. 815-831.

[16] W. Hrusa, J. Nohel, and M. Renardy, Mathematical Problems in Viscoelasticity, no. 35 in Pitman Monographs and Surveys in Pure and Applied Mathematics, Longman Scientific \& Technical, 1987.

[17] D. D. Joseph and Y. Y. Renardy, Fundamentals of two-fluid dynamics. Part I, SpringerVerlag, New York, 1993. Mathematical theory and applications.

[18] — Fundamentals of two-fluid dynamics. Part II, Springer-Verlag, New York, 1993. Lubricated transport, drops and miscible liquids.

[19] S. N. KRuzKov, First order quasilinear equations in several independent variables, Math. USSR Sbornik, 10 (1970), pp. 217-243.

[20] J. Li and Y. Renardy, Numerical study of flows of two immiscible liquids at low reynolds number, SIAM Review, 42 (2000), pp. 417-439.

[21] J. L. Lions, On some questions in boundary value problems of mathematical physics, in Contemporary developments in continuum mechanics and partial differential equations: proceedings of the International Symposium on Continuum Mechanics and Partial Differential Equations, Rio de Janeiro, G. M. de la Penha and L. A. J. Medeiros, eds., vol. 30 of Mathematics Studies, New York, 1977, North-Holland, pp. 285-346. 
[22] P. L. Lions, Mathemaitcial Topics in Fluid Mechanics, Volume 1: Incompressible Models, Oxford Press, Oxford, U.K., 1996.

[23] C. Liu, Dynamic theory for incompressible smectic-A liquid crystals: Existence and regularity, Discrete and Continuous Dynamical Systems, 6 (2000), pp. 591-608.

[24] J. Lowengrub And L. Truskinovsky, Quasi-icompressible Cahn-Hilliard fluid and topological transitions, Proc. Royal Society London, 454 (1998), pp. 2617-2654.

[25] F. Отto And W. E, Thermodynamically driven incompressible fluid mixtures, J. Chemical Physics, 107 (1997), pp. 10177-10184.

[26] C. S. Peskin And C. Tu, Stability and instability in the computation of flows with moving immersed boundaries: a comparison of three methods, SIAM J. Sci. Statist. Comput., 13 (1992), pp. 1361-1376.

[27] J. Rubinstein, P. Sternberg, and J. B. Keller, Fast reaction, slow diffusion, and curve shortening, SIAM J. Appl. Math., 49 (1989), pp. 116-133.

[28] _ Reaction-diffusion processes and evolution to harmonic maps, SIAM J. Appl. Math., 49 (1989), pp. 1722-1733.

[29] R. E. Showalter, Monotone operators in Banach space and nonlinear partial differential equations, American Mathematical Society, Providence, RI, 1997.

[30] L. TARTAR, Compensated compactness and applications to partial differential equations, in Research Notes in Mathematics, Nonlinear Analysis, and Mechanics, Heriot Watt Symposium, R. J. Knops, ed., vol. 4, Pitman Press, 1979.

[31] R. Temam, Navier-Stokes Equations, North Holland, 1977. 